\title{
KNESER'S THEOREM FOR WEAK SOLUTIONS \\ OF AN INTEGRAL EQUATION \\ WITH WEAKLY SINGULAR KERNEL
}

\section{Aldona Dutkiewicz and Stanisław Szufla}

\author{
ABSTRACT. We prove that the set of all weak solutions of the Volterra integral \\ equation (1) is nonempty, compact and connected.
}

Assume that $D=[0, a]$ is a compact interval in $\mathbb{R}, E$ is a sequentially weakly complete Banach space, $B=\{x \in E:\|x\| \leqslant b\}$. We prove the existence of a weak solution of the integral equation

$$
x(t)=\int_{0}^{t} K(t, s) f(s, x(s)) d s,
$$

where

$1^{\circ} f: D \times B \mapsto E$ is a weakly-weakly continuous function such that $\|f(t, x)\| \leqslant M$ for $(t, x) \in D \times B$;

$2^{\circ} K(t, s)=\frac{H(t, s)}{(t-s)^{r}}, 0<r<1$, where $H$ is a real continuous function.

Moreover, we study the topological structure of the set of all weak solutions of (1). In what follows we shall need the following result of W. Mydlarczyk given in [6].

THEOREM 1. Let $\alpha>0$ and let $g: \mathbb{R}_{+} \mapsto \mathbb{R}_{+}$be a nondecreasing function such that $g(0)=0, g(t)>0$ for $t>0$. Then the equation

$$
u(t)=\int_{0}^{t}(t-s)^{\alpha-1} g(u(s)) d s \quad(t \geqslant 0)
$$

has a nontrivial continuous solution if and only if

$$
\int_{0}^{\delta} \frac{1}{s}\left[\frac{s}{g(s)}\right]^{1 / \alpha} d s<\infty \quad(\delta>0) .
$$

Let $c=\max _{t, s \in D}|H(t, s)|$. Choose a positive number $d$ such that $d \leqslant a$ and $M \cdot c \cdot \frac{d^{1-r}}{1-r}<b$. Denote by $L=M \cdot c \cdot \frac{d^{1-r}}{1-r}$. Hence $L<b$.

2000 Mathematics Subject Classification: Primary 45N05. 
Let $J=[0, d]$. Denote by $C_{w}(J, E)$ the space of weakly continuous functions $J \mapsto E$ endowed with the topology of weak uniform convergence. Moreover, denote by $\beta$ the measure of weak noncompactness introduced by De Blasi [2].

Let us recall that for any nonvoid, bounded subset $A$ of a Banach space $E$, $\beta(A)=\inf \{\varepsilon>0:$ there exists a weakly compact set $K$ such that $A \subset K+\varepsilon B\}$, where $B$ is the norm unit ball. Recall that $\beta$ has the following properties:

$1^{\circ} A \subset B \Rightarrow \beta(A) \leqslant \beta(B)$;

$2^{\circ} \beta\left(\bar{A}^{w}\right)=\beta(A)$, where $\bar{A}^{w}$ denotes the weak closure of $A$;

$3^{\circ} \beta(A)=0 \Leftrightarrow \bar{A}^{w}$ is weakly compact;

$4^{\circ} \beta(A \cup B)=\max (\beta(A), \beta(B))$;

$5^{\circ} \beta(\operatorname{conv} A)=\beta(A)$;

$6^{\circ} \beta(A+B) \leqslant \beta(A)+\beta(B)$

$7^{\circ} \beta(\lambda A)=|\lambda| \beta(A),(\lambda \in \mathbb{R})$;

$8^{\circ} \beta\left(\bigcup_{|\lambda| \leqslant h} \lambda A\right)=h \beta(A)$.

Let $V$ be a subset of $C_{w}(J, E)$. Put $V(t)=\{u(t): u \in V\}$ and $V(T)=\{u(t)$ : $u \in V, t \in T\}$. Let us recall the well known Ambrosetti type

LEMMA 1. If the set $V$ is strongly equicontinuous and uniformly bounded, then

(a) the function $t \mapsto \beta(V(t))$ is continuous on $J$;

(b) for each compact subset $T$ of $J$ one has $\beta(V(T))=\sup \{\beta(V(t)): t \in T\}$.

Let $\tilde{B}$ denote the set of all weakly continuous functions $J \mapsto B$. We shall consider $\tilde{B}$ as a topological subspace of $C_{w}(J, E)$. Put

$$
F(x)(t)=\int_{0}^{t} K(t, s) f(s, x(s)) d s, \quad(x \in \tilde{B}, t \in J) .
$$

Arguing similarly as in [4, p. $132-133]$ we can prove that the set $F(\tilde{B})$ is strongly equicontinuous. On the other hand, from the following Krasnoselskil type

LEMma 2. For any $\varphi \in E^{*}, \varepsilon>0$ and $z \in \tilde{B}$ there exists a weak neighbourhood $U$ of 0 in $E$ such that $|\varphi(f(t, z(t))-f(t, w(t)))| \leqslant \varepsilon$ for $t \in J$ and $w \in \tilde{B}$ such that $w(s)-z(s) \in U$ for all $s \in J$. [8]

It follows that $F$ is a continuous mapping from $\tilde{B}$ into $C_{w}(J, E)$.

For given $\varepsilon>0$ denote by $S_{\varepsilon}$ the set of all $z \in \tilde{B}$ such that $\|z(t)-F(z)(t)\|<\varepsilon$ for all $t \in J$.

LEMMA 3. For each $\varepsilon, 0<\varepsilon<b-L$, the set $S_{\varepsilon}$ is nonempty and connected in $C_{w}(J, E)$.

Proof. For any positive integer $n$ we define $F_{n}(x)(t)=F(x)\left(r_{n}(t)\right)(x \in \tilde{B}$, $t \in J)$, where

Put

$$
r_{n}(t)= \begin{cases}0, & \text { if } 0 \leqslant t \leqslant d / n \\ t-d / n, & \text { if } d / n \leqslant t \leqslant d\end{cases}
$$

$$
\sup _{t \in J, x \in \tilde{B}}\left\|F(x)(t)-F(x)\left(r_{n}(t)\right)\right\|=w_{n} .
$$


Because the set $F(\tilde{B})$ is equicontinuous, we have $w_{n} \mapsto 0$ as $n \mapsto \infty$. Moreover, there exists a unique $z_{n} \in \tilde{B}$ such that $z_{n}=F_{n}\left(z_{n}\right)$. It is clear from (2) that $z_{n} \in S_{\varepsilon}$ for sufficiently large $n$. Fix $u_{0}, u_{1} \in S_{\varepsilon}$. Put

$$
\eta=\max \left(\sup _{t \in J}\left\|u_{0}(t)-F\left(u_{0}\right)(t)\right\|, \sup _{t \in J}\left\|u_{1}(t)-F\left(u_{1}\right)(t)\right\|\right)
$$

and $\delta=\varepsilon-\eta$. Fix a positive integer $n$ such that $2 w_{n}<\delta$. Let

$$
a_{\lambda}=\lambda\left(u_{1}-F_{n}\left(u_{1}\right)\right)+(1-\lambda)\left(u_{0}-F_{n}\left(u_{0}\right)\right) \text { for } 0 \leqslant \lambda \leqslant 1 .
$$

It follows from (2) that

$$
\begin{array}{r}
\left\|u_{i}(t)-F_{n}\left(u_{i}\right)(t)\right\| \leqslant\left\|u_{i}(t)-F\left(u_{i}\right)(t)\right\|+\left\|F\left(u_{i}\right)(t)-F_{n}\left(u_{i}\right)(t)\right\| \leqslant \eta+w_{n} \\
(i=0,1) .
\end{array}
$$

Hence

$$
\left\|a_{\lambda}(t)\right\| \leqslant \eta+w_{n} \text { for } t \in J \text { and } 0 \leqslant \lambda \leqslant 1 .
$$

Arguing similarly as in $[8$, p. 122$]$ we can prove that for each $\lambda \in[0,1]$ there exists a unique $u_{\lambda}$ such that $u_{\lambda}=a_{\lambda}+F_{n}\left(u_{\lambda}\right)$ and $u_{\lambda}$ depends continuosly on $\lambda$. Since

$$
\int_{0}^{t} \frac{d s}{(t-s)^{r}}=\frac{t^{1-r}}{1-r}
$$

we have

$$
\|F(x)(t)\|=\left\|\int_{0}^{t} K(t, s) f(s, x(s)) d s\right\| \leqslant c \cdot M \cdot \frac{d^{1-r}}{1-r}=L \text { for } x \in \tilde{B}, t \in J .
$$

From this and inequalities (2)-(3) we obtain

$$
\left\|u_{\lambda}(t)\right\| \leqslant\left\|a_{\lambda}(t)\right\|+\left\|F\left(u_{\lambda}\right)\left(r_{n}(t)\right)\right\| \leqslant \eta+w_{n}+L<\eta+\delta+L=\varepsilon+L<b
$$

and

$$
\begin{aligned}
\left\|u_{\lambda}(t)-F\left(u_{\lambda}\right)(t)\right\| & =\left\|a_{\lambda}(t)+F_{n}\left(u_{\lambda}\right)(t)-F\left(u_{\lambda}\right)(t)\right\| \\
& \leqslant\left\|a_{\lambda}(t)\right\|+\left\|F_{n}\left(u_{\lambda}\right)(t)-F\left(u_{\lambda}\right)(t)\right\| \\
& \leqslant \eta+2 w_{n}<\eta+\delta=\varepsilon \quad(t \in J, 0 \leqslant \lambda \leqslant 1),
\end{aligned}
$$

so that $u_{\lambda} \in S_{\varepsilon}$. From this we conclude that for any $u_{0}, u_{1} \in S_{\varepsilon}$ there exists a continuous curve in $S_{\varepsilon}$ connecting $u_{0}$ and $u_{1}$, which proves that $S_{\varepsilon}$ is arcwise connected.

The main result of the paper is the following

THEOREM 2. Let $g: \mathbb{R}_{+} \mapsto \mathbb{R}_{+}$be a continuous nondecreasing function such that $g(0)=0, g(t)>0$ for $t>0$ and

$$
\int_{0}^{\delta} \frac{1}{s}\left[\frac{s}{g(s)}\right]^{1 /(1-r)} d s=\infty \quad(\delta>0)
$$

If $1^{\circ}$ and $2^{\circ}$ hold and

$$
\beta(f(J \times X)) \leqslant g(\beta(X)) \text { for } X \subset B,
$$


then the set $S$ of all weak solutions of (1) defined on $J$ is nonempty, compact and connected in $C_{w}(J, E)$.

Proof. 1. First we shall show that the set $S$ is nonempty. By Lemma 3 there exists a sequence $\left(u_{n}\right)$ such that $u_{n} \in \tilde{B}$ and

$$
\lim _{n \rightarrow \infty} \sup _{t \in J}\left\|u_{n}(t)-F\left(u_{n}\right)(t)\right\|=0 .
$$

Let $V=\left\{u_{n}: n \in N\right\}$. Since

$$
\begin{gathered}
V \subset\left\{u_{n}-F\left(u_{n}\right): n \in N\right\}+F(V) \\
V(t) \subset\left\{u_{n}(t)-F\left(u_{n}\right)(t): n \in N\right\}+F(V)(t), \\
F(V)(t) \subset V(t)-\left\{u_{n}(t)-F\left(u_{n}\right)(t): n \in N\right\},
\end{gathered}
$$

it follows from (6) that the set $V$ is strongly equicontinuous and

$$
\beta(V(t))=\beta(F(V)(t)) \text { for } t \in J \text {. }
$$

Hence, by Lemma 1, the function $t \mapsto v(t)=\beta(V(t))$ is continuous on $J$.

Fix $t \in J$ and $\varepsilon>0$, and choose $\eta>0$ such that

$$
\left\|\int_{t-\eta}^{t} \frac{H(t, s)}{(t-s)^{r}} f(s, x(s)) d s\right\| \leqslant \int_{t-\eta}^{t} \frac{|H(t, s)|}{(t-s)^{r}} M d s<\varepsilon \text { for all } x \in \tilde{B} .
$$
From the continuity of the function $\frac{H(t, s)}{(t-s)^{r}} g(v(s))$ on $[0, t-\eta]$ it follows that there
exists $\delta>0$ such that

$$
\left|\frac{H(t, \tau)}{(t-\tau)^{r}} g(v(q))-\frac{H(t, s)}{(t-s)^{r}} g(v(s))\right|<\varepsilon
$$

if $|\tau-s|<\delta,|q-s|<\delta, q, s, \tau \in[0, t-\eta]$. Divide the interval [0,t- $\eta]$ into $n$ parts $0=t_{0}<t_{1}<\ldots<t_{n}=t-\eta$ so that $\Delta t_{i}=t_{i}-t_{i-1}<\delta(i=1, \ldots, n)$. Put $T_{i}=\left[t_{i-1}, t_{i}\right]$. By Lemma 1 for each $i$ there exists $s_{i} \in T_{i}$ such that $\beta\left(V\left(T_{i}\right)\right)=v\left(s_{i}\right)$ $(i=1, \ldots, n)$. Put

$$
\int_{T} \frac{H(t, s)}{(t-s)^{r}} f(s, V(s)) d s=\left\{\int_{T} \frac{H(t, s)}{(t-s)^{r}} f(s, x(s)) d s: x \in V\right\} .
$$

Because

$$
\begin{aligned}
\int_{0}^{t-\eta} \frac{H(t, s)}{(t-s)^{r}} f(s, V(s)) d s & \subset \sum_{i=1}^{n} \int_{t_{i-1}}^{t_{i}} \frac{H(t, s)}{(t-s)^{r}} f(s, V(s)) d s \\
& \subset \sum_{i=1}^{n} \Delta t_{i} \overline{\operatorname{conv}}\left\{\frac{H(t, s)}{(t-s)^{r}} f(s, x(s)): s \in T_{i}, x \in V\right\},
\end{aligned}
$$


we get

$$
\begin{aligned}
\beta & \left(\int_{0}^{t-\eta} \frac{H(t, s)}{(t-s)^{r}} f(s, V(s)) d s\right) \\
& \leqslant \sum_{i=1}^{n} \Delta t_{i} \beta\left(\overline{\operatorname{conv}}\left\{\frac{H(t, s)}{(t-s)^{r}} f(s, x(s)): s \in T_{i}, x \in V\right\}\right) \\
& =\sum_{i=1}^{n} \Delta t_{i} \beta\left(\left\{\frac{H(t, s)}{(t-s)^{r}} f(s, x(s)): s \in T_{i}, x \in V\right\}\right) \\
& =\sum_{i=1}^{n} \Delta t_{i} \max _{s \in T_{i}} \frac{|H(t, s)|}{(t-s)^{r}} \beta\left(f\left(J \times V\left(T_{i}\right)\right)\right. \\
& \leqslant \sum_{i=1}^{n} \Delta t_{i} \frac{\left|H\left(t, \tau_{i}\right)\right|}{\left(t-\tau_{i}\right)^{r}} g\left(\beta\left(V\left(T_{i}\right)\right)\right) \leqslant \sum_{i=1}^{n} \Delta t_{i} \frac{\left|H\left(t, \tau_{i}\right)\right|}{\left(t-\tau_{i}\right)^{r}} g\left(v\left(s_{i}\right)\right) .
\end{aligned}
$$

Here $\tau_{i} \in T_{i}$ is a number such that $\frac{\left|H\left(t, \tau_{i}\right)\right|}{\left(t-\tau_{i}\right)^{r}}=\max _{s \in T_{i}} \frac{|H(t, s)|}{(t-s)^{r}}$. Furthermore, from

$$
\sum_{i=1}^{n} \Delta t_{i} \frac{\left|H\left(t, \tau_{i}\right)\right|}{\left(t-\tau_{i}\right)^{r}} g\left(v\left(s_{i}\right)\right) \leqslant \int_{0}^{t-\eta} \frac{|H(t, s)|}{(t-s)^{r}} g(v(s)) d s+\varepsilon(t-\eta) .
$$

Since

$$
F(V)(t) \subset \int_{0}^{t-\eta} \frac{H(t, s)}{(t-s)^{r}} f(s, V(s)) d s+\int_{t-\eta}^{t} \frac{H(t, s)}{(t-s)^{r}} f(s, V(s)) d s
$$

and

$$
\beta\left(\left\{\int_{t-\eta}^{t} \frac{H(t, s)}{(t-s)^{r}} f(s, x(s)) d s: x \in V\right\}\right) \leqslant 2 \varepsilon,
$$

from inequalities (8) and (9) it follows that

$$
\begin{aligned}
\beta(F(V)(t)) & \leqslant \int_{0}^{t-\eta} \frac{|H(t, s)|}{(t-s)^{r}} g(v(s)) d s+\varepsilon(t-\eta)+2 \varepsilon \\
& \leqslant \int_{0}^{t} \frac{|H(t, s)|}{(t-s)^{r}} g(v(s)) d s+\varepsilon t+2 \varepsilon .
\end{aligned}
$$

As the last inequality is satisfied for every $\varepsilon>0$, we get

$$
\beta(F(V)(t)) \leqslant \int_{0}^{t} \frac{|H(t, s)|}{(t-s)^{r}} g(v(s)) d s
$$

Therefore, by (7),

i.e.,

$$
\beta(V(t)) \leqslant \int_{0}^{t} \frac{|H(t, s)|}{(t-s)^{r}} g(v(s)) d s
$$

$$
v(t) \leqslant \int_{0}^{t} \frac{|H(t, s)|}{(t-s)^{r}} g(v(s)) d s \text { for } t \in J .
$$


Applying Theorem 1 with $\alpha=1-r$ and theorem on integral inequalities [3, Lemma 1] from this we deduce that $v(t)=0$ for $t \in J$. Therefore, by Lemma 1

$$
\beta(V(J))=\sup \{\beta(V(t)): t \in J\}=0
$$

i.e., $V$ is relatively compact in $C_{w}(J, E)$. Hence we can find a subsequence $\left(u_{n_{k}}\right)$ of $\left(u_{n}\right)$ which converges in $C_{w}(J, E)$ to a limit $u$. As $F$ is continuous, from this and (6) we conclude that $u=F(u)$. This proves that the set $S$ is nonempty.

2. Further, since $F$ is continuous, $S$ is closed in $C_{w}(J, E)$. As $S=F(S)$, we have $\beta(S(t))=\beta(F(S)(t))$ for $t \in J$. Arguing similarly as in 1, we can show that $S$ is compact in $C_{w}(J, E)$.

Now we shall prove that $S$ is connected. Suppose that $S$ is not connected in $C_{w}(J, E)$. As $S$ is compact, there are nonempty compact sets $S_{1}, S_{2}$ such that $S=S_{1} \cup S_{2}$ and $S_{1} \cap S_{2}=\emptyset$, and there are two disjoint open sets $U_{1}, U_{2}$ such that $S_{1} \subset U_{1}, S_{2} \subset U_{2}$. Let $U=U_{1} \cup U_{2}$. We choose $n_{0}$ such that $1 / n_{0}<b-L$. Suppose that for each $n \geqslant n_{0}$ there exists $u_{n} \in S_{1 / n} \backslash U$. Put $V=\left\{u_{n}: n \in N\right\}$. Because $\lim _{n \rightarrow \infty} \sup _{t \in J}\left\|u_{n}(t)-F\left(u_{n}\right)(t)\right\|=0$, by repeating the argument from 1 we can prove that there exists $u_{0} \in \bar{V}$ such that $u_{0}=F\left(u_{0}\right)$, i.e., $u_{0} \in S$. Furthermore, $\bar{V} \subset C_{w}(J, E) \backslash U$, as $U$ is open, so that $u_{0} \in S \backslash U$, a contradiction. Therefore there exists $k \in N$ such that $S_{1 / k} \subset U$. Since $U_{1} \cap S_{1 / k} \neq \emptyset \neq U_{2} \cap S_{1 / k}$, this shows that $S_{1 / k}$ is not connected, which contradicts Lemma 3. Hence $S$ is connected.

The authors express their appreciation to the referee for his suggestions to amend an earlier version of the paper.

\section{References}

[1] J. Banaś, K. Goebel, Measure of noncompactness in Banach spaces, Marcel Dekker, New York-Basel, 1980.

[2] F. S. De Blasi, On a property of the unit sphere in a Banach space, Bull. Math. Soc. Sci. Math. R. S. Roumanie 21 (1997), 259-262.

[3] G. Gripenberg, On the uniqueness of solutions of Volterra equations, J. Integral Eqns Appl. $2: 3$ (1990), 421-430.

[4] J. Januszewski, On Volterra integral equations with weakly sigular kernel in Banach spaces, Demonstr. Math. 26 (1993), 131-136.

[5] Z. Kubáček, On the structure of fixed point sets of some compact maps in the Fréchet space, Math. Bohem. 118 (1993), 343-358.

[6] W. Mydlarczyk, The existence of nontrivial solutions of Volterra equations, Math. Scand. 68 (1991), 83-88.

[7] V.Šeda, $R_{\delta}$-set of solutions to a boundary value problem, Topol. Methods Nonlinear Anal. $16: 1(2000), 93-101$.

[8] S. Szufla, Sets of fixed points of nonlinear mappings in function spaces, Funkcialaj Ekvacioj 22 (1979), 121-126.

[9] S. Szufla, Kneser's theorem for weak solutions of an mth-order ordinary differential equation in Banach spaces, Nonlinear Anal. 38 (1999), 785-791.

Faculty of Mathematics and Computer Science

(Received 2002 2004) Adam Mickiewicz University

(Revised 2001 2005)

Umultowska 87

61-614 Poznań

Poland 